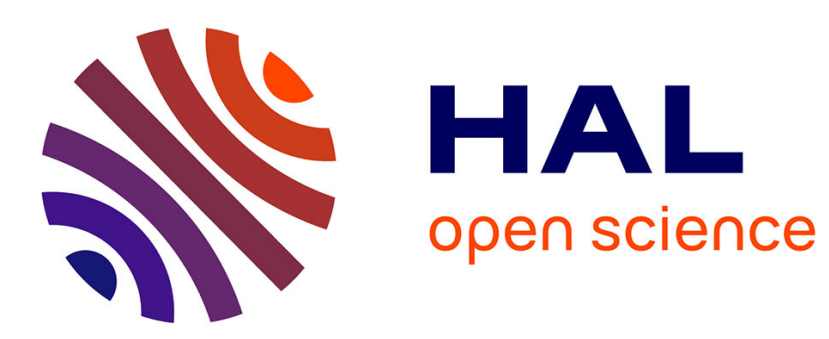

\title{
An experimental study on the influence of the coordination number on grain crushing
}

\author{
Younes Salami, Christophe Dano, Pierre-Yves Hicher
}

\section{To cite this version:}

Younes Salami, Christophe Dano, Pierre-Yves Hicher. An experimental study on the influence of the coordination number on grain crushing. European Journal of Environmental and Civil Engineering, 2019, 23 (3), pp.432-448. 10.1080/19648189.2017.1285251 . hal-02007127

\section{HAL Id: hal-02007127 \\ https://hal.science/hal-02007127}

Submitted on 24 Oct 2019

HAL is a multi-disciplinary open access archive for the deposit and dissemination of scientific research documents, whether they are published or not. The documents may come from teaching and research institutions in France or abroad, or from public or private research centers.
L'archive ouverte pluridisciplinaire HAL, est destinée au dépôt et à la diffusion de documents scientifiques de niveau recherche, publiés ou non, émanant des établissements d'enseignement et de recherche français ou étrangers, des laboratoires publics ou privés. 


\title{
An experimental study on the influence of the coordination number on grain crushing
}

\author{
Younes Salami $^{\mathrm{a} *}$, Christophe Dano ${ }^{\mathrm{b}}$ and Pierre-Yves Hicher ${ }^{\mathrm{a}}$ \\ ${ }^{a}$ GeM (CNRS UMR 6183), Ecole Centrale de Nantes, LUNAM University, Nantes Cedex, France; \\ ${ }^{b}$ 3SR (CNRS UMR 5521), Université Grenoble Alpes, Grenoble, France
}

\begin{abstract}
In soil mechanics, the material behaviour is significantly affected by grain crushing. This energy dissipating mechanism, along with the frictional rearrangement of grains, is responsible for the energy loss associated with plasticity. However, the micromechanical factors at the grain scale have not been studied by constitutive models which tend to focus on macroscopic parameters. In this study, the influence of the coordination number on the fragmentation of a single cylindrical grain specimen has been examined with a new device by which a series of multipoint crushing tests has been conducted. Experiments conducted with this device have shown the importance of the contacts number, position, type and force in the fragmentation of the individual grain. The results, analysed by imaging techniques, demonstrate that the existing models treating grain rupture are indeed incapable of reproducing the observed fragmentation. Two types of cracks were distinguished, each corresponding to a different crack mode, depending on the contact arrangement. The results of this study could be integrated into fragmentation models for predicting the occurrence of cracks, and the shape of the resulting fragments.
\end{abstract}

Keywords: grain crushing test; coordination number; fracture mode

\section{Introduction}

Soil behaviour at high stresses is highly dependent on the evolution of the grain size distribution because the occurrence of significant grain crushing gives rise to the evolution of soil properties such as compressibility, dilatancy and shear strength. In order to estimate this effect, a study at the grain scale must be considered, and the grain characteristics and interactions analysed. Particle strength, which is the primary property affecting the fracture of a grain, has been extensively investigated (Jaeger, 1967) through diametric compression tests and is usually expressed from a probabilistic point of view by assuming a Weibull's distribution (McDowell \& Amon, 2000). However, a secondary effect, which should equally affect particle crushing, is the coordination number. It is commonly accepted that larger particles have a higher coordination number due to their size, which could partially compensate for their weaker strength. Smaller particles however, albeit stronger, have a higher probability of finding themselves under a set of two diametrically opposed forces (i.e. low coordination number), which is the most favourable case for fracture. The competing effect of these two processes continues during loading, and stabilizes when the final grain size distribution is reached. If one of

*Corresponding author. Email: younes.salami@ec-nantes.fr 
the effects becomes dominant, the grain sizes will become uniform, and the ultimate distribution will consist of uniformly sized fines. In reality, the ultimate grain size distribution is fractal (McDowell, Bolton, \& Robertson, 1996), which means that both the size of the grain and its coordination number remain active during the breakage process. This effect has been noted by several authors (Auvinet \& Marsal, 1975; McDowell \& Bolton, 1998; Tsoungui, Vallet, Charmet, \& Roux, 1999), but to date, very few experimental investigations have been conducted to study this effect.

Todisco, Coop and Guo (2015) constructed an experimental device to study multiparticle crushing, where one particle of sand or limestone was crushed between a set of steel balls or particles glued to the loading frame. By means of Weibull's statistics, they confirmed that smaller grains are usually stronger and that a higher coordination number resulted in a decreased probability of crushing.

Numerically, two failure criteria have been suggested for a two dimensional grain subjected to a random distribution of contact forces. The first one, suggested by (Tsoungui, Vallet, \& Charmet, 1998, 1999; Tsoungui, Vallet, Charmet, et al., 1999) replaces the distribution of forces by a set of two orthogonal diametrically opposed forces, $F_{\max }$ and $F_{\min }$, inclined by an angle $\Phi$, in the form of an inclined cross (Figure 1(b)). This configuration is equivalent, from a mechanical point of view, to the real distribution of contact forces, represented in Figure 1(a). After stating that the fracture occurs in a tensile splitting mode, a series of finite element (FEM) computations was performed, and the stresses near the centre of the disk where the crack was supposed to originate were analysed, which led to a failure criterion. This criterion, although limited, suggested that the configurations closest to the diametrically opposed set of forces resulted in a higher probability of rupture and that the lowest probability of rupture corresponded to a grain under hydrostatic pressure.

The second failure criterion, developed by Ben-Nun and Einav (2010) was based on Discrete Elements Models (DEM) simulations performed by Sukumaran, Einav, and Dyskin (2006), which was numerically applied to uniaxial compression, showing that the ultimate grain size distribution was fractal. This model assumed that cracks resulted from an in-plane shear fracture mode (mode II) (Figure 1(c)). Based on this fragmentation model, a grain under an isotropic loading configuration (i.e. when subjected to a uniform pressure or when the contact forces are diametrically opposed) could break, which was impossible with the use of Tsoungui's model. An average of the normal

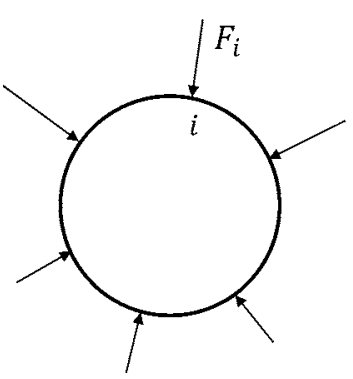

(a)

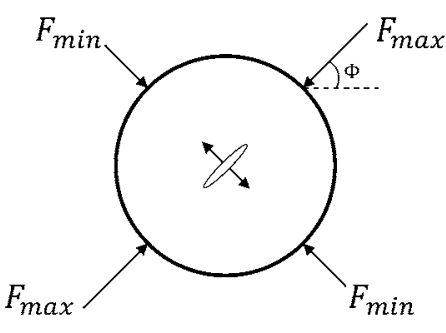

(b)

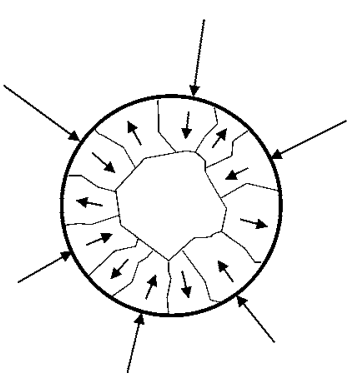

(c)

Figure 1. Failure criteria, according to (b) Tsoungui et al. (1999) and (c) Ben-Nun and Einav (2010) compared to reference case (a). 
components of the contact forces was compared to a critical value based on a formula suggested by Sukumaran et al. (2006).

According to the numerical study by Sukumaran et al., the effect of the curvature and the effect of the coordination number on the critical force are considered through two multiplicative factors $f_{D}$ and $f_{C N}$, respectively. The critical force $F_{C}$ is written:

$$
F_{c}=d^{2} r_{c} f_{D} f_{C N}
$$

With $\sigma_{C}$ being the critical tensile stress. The natural variability in the strengths of the specimens could eventually be represented using Weibull statistics by including an additional factor for this effect. This formula includes the effects of the coordination number through an exponential reduction factor $f_{C N}$ :

$$
f_{C N}=(C-1) \exp \left(\left(\frac{D}{d}\right) \frac{(C-2)(C-3)}{4 C}\right)
$$

with $C$ being the coordination number, $d$ the diameter of the crushed grain and $D$ the diameter of the surrounding particles.

\section{The experimental procedure}

\section{The device}

A novel apparatus for the simulation of the effects of the coordination number on the fragmentation of a single cylindrical grain specimen was designed (Figure 2). It consists of a round metal mounting frame, stiff enough not to be deformed by the applied range of loads. The specimen is placed on the bottom platen, so that its centre coincides with the centre of the frame. A removable element permits the specimen to be aligned with the load cell vertical axis. A rigid stainless steel plate, screwed to the load cell and supporting two displacement transducers out of plane (not represented in Figure 2), is fixed on the top part of the specimen.
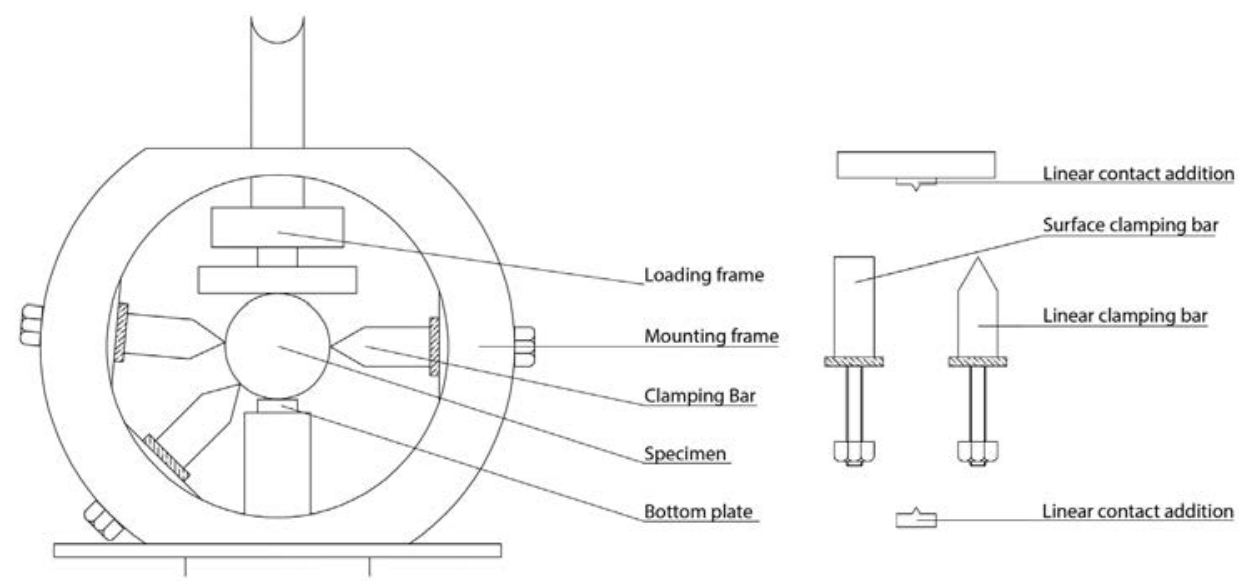

Figure 2. Scheme of the apparatus (left) and of the two types of clamping bars and the linear contact additions (right) (Salami, Dano, Hicher, Colombo, \& Denain, 2015). 
The clamping bars are then bolted along the lateral circular surface of the device, locking the specimen in the desired contact configuration. The bars can be moved freely around the lateral surface of the specimen. In most of the experiments, the clamping bars were just put in contact with the specimen, in order to induce a null lateral displacement condition: the lateral contacts are then passive. In other tests, the clamping bars were pre-stressed before applying the vertical load: the lateral contacts behave like active contacts similarly to the contacts in the vertical loading direction.

Moreover, in order to study the effect of the contact type on the grain crushing, two types of clamping bars were manufactured. The contact can be either linear or along a surface. The whole device is then placed on a loading frame, and the specimen is crushed under a particular set of contacts, following a displacement-controlled mode with a displacement rate of $0.5 \mathrm{~mm} / \mathrm{min}$. The loading frame delivers a force up to 50 $\mathrm{kN}$ and the force and displacements are monitored using a load cell with a resolution of $0.06 \mathrm{~N}$ and displacement transducers with a resolution of $0.01 \mathrm{~mm}$. However, because of the acquisition rate ( 1 acq. per sec.), the absolute uncertainty in the maximum force is estimated to be about $0.15 \mathrm{kN}$.

The present device is different from the one used by Todisco et al. (2015), in which the lateral contacts were passive, and the force applied during loading was radial, and not along the direction of loading (since the contacting grains were glued to the loading frame). Moreover, our device allows us to better reproduce the case of a polydisperse granular material, where the coordination number is usually high and where the distribution of contacts along the lateral surface is random. The nature of the contacts, which are either along a surface or along a line, can represent the contact with either a bigger particle (contact along a surface or face-to-face contact mode) or a smaller one (contact along a line or edge-to-face contact mode).

\section{The materials tested}

The tests were first performed on natural rock specimens but, due to their variability and heterogeneity, the results contained too much scatter that we were compelled to work with a more controllable material. The working materials preferred were a mortar and a cement mix, but the experiments presented in this paper only concern the mortar. The cylindrical specimens had a diameter of $52 \mathrm{~mm}$ and a width of $21 \mathrm{~mm}$. They were prepared in accordance with the French standard NF EN 196-1 (2006) for batches of 15 units. The cement used was a CEM1 52.5R, and it was mixed with a Hostun siliceous sand HN 0.6/1.6. The water to cement ratio was 0.5. As for the curing conditions, the disks were set up in a humidity chamber $(100 \%$ relative humidity, and a temperature of $18{ }^{\circ} \mathrm{C}$ ). By controlling every step of the manufacturing process of the disks, we were able to maintain the standard deviation for the values of the critical force distributions around $0.5 \mathrm{kN}$ for the diametric compression tests and around $0.6 \mathrm{kN}$ for most of the other configurations.

Cylindrical specimens, representing a basic 2D simulation of a disk, were also well adapted for Digital Image Correlation (DIC) observations.

\section{Digital image correlation}

DIC is an optical experimental technique which allows surface displacements to be measured. This technique has been theoretically and experimentally validated and has become in recent years a robust and accurate method in experimental mechanics. Using 
a digital camera, images were taken in regular intervals and the strains were computed from the displacements measured using the continuum mechanics theory. For the purpose of our tests, a progressive CCD camera (AVT Manta G-504 B) with a resolution of $2452 \times 2056$ pixels delivering 256 levels of grey was used. The camera was placed at $50 \mathrm{~cm}$ from the specimen and a photo was taken every second. For this resolution and location, one pixel represents a $32 \mu \mathrm{m}$ square surface element. The DIC analysis was performed with the software VIC-2D ${ }^{\mathrm{TM}}$ (Correlated Solutions, 2009), which computes displacements with an accuracy of $1 / 20$ pixel, which corresponds to $\pm 1.6 \mu \mathrm{m}$ for our resolution. The accuracy for the strain measurements is approximately 50 microstrain $(0.005 \%)$. The open source program nCorr (Blaber, Adair, \& Antoniou, 2015) was used to determine and sketch the principal strain distributions.

The DIC tests were performed according to three stages:

- The surface of the specimen was prepared in order to maximise the contrast of the images taken by the camera and to facilitate the processing. This step consists of painting a black and white pattern on the surface, adjusting the light, and adjusting the camera accordingly.

- The image was acquired by the camera and a separate computer. The images are in the pgm format (portable greymap file format).

- The post treatment used the VIC-2D or the nCorr software. A region of interest (ROI) was defined which the software decomposes into elemental meshes. The shape and size of these elements characterise the correlation since DIC consists of a comparison between the initial and the deformed element. Once the deformations and the rotations are defined, fields of deformations and rotations are extrapolated along the ROI. The data, which is expressed in terms of pixels, is converted to millimetres after a calibration process.
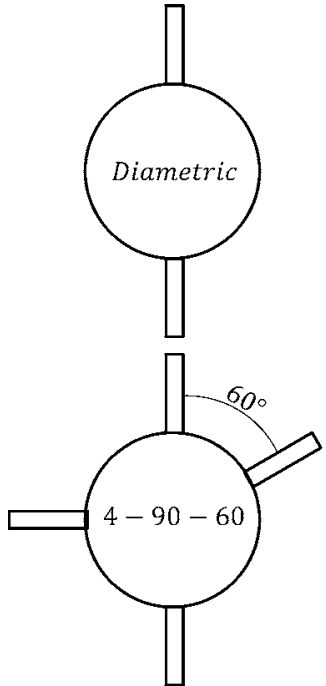
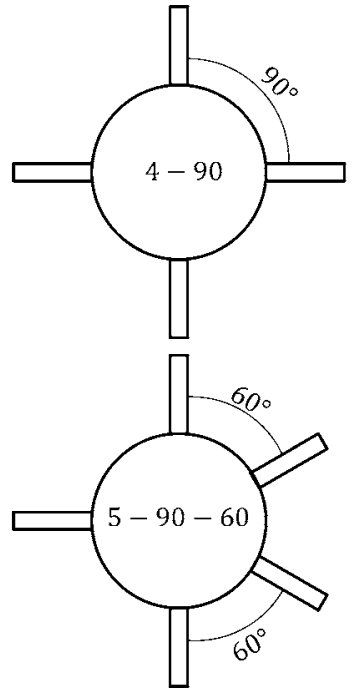
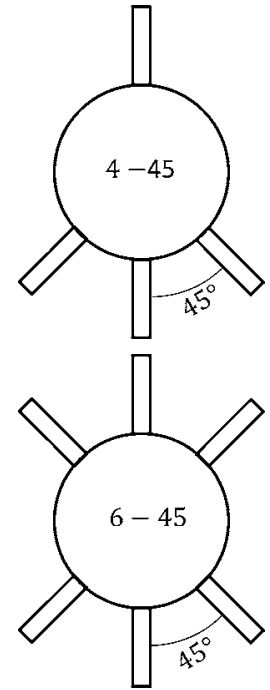

Figure 3. The six contact configurations tested. 


\section{Experimental program}

The results presented herein correspond to six contact configuration (Figure 3). The varying coordination number allowed us to study its effect on the crushing of the specimen, whereas the contact position effect was studied by moving the clamping bars along the lateral surface. The diametric configuration, the 4-90, 4-45, 4-90-60, 5-90-60 and the 6-45 configurations are named in order to represent the coordination number (the first number of the name) and the angles between the secondary contacts and the vertical ones.

Fifteen batches, each containing 15 specimens, were prepared, with the aim of testing each configuration of Figure 3: one contact configuration is tested on one batch of 15 specimens. A batch is prepared one week before beginning the experiments, since mortar is sufficiently hard at 7 days to exhibit brittle fracture when crushed. At least 3 specimens per batch were systematically tested in diametric conditions, so that a reference for subsequent comparison could be provided.

\section{Test results}

\section{General results}

Table 1 summarises the results of the crushing tests. 15 series of tests corresponding to the configurations presented in Figure 3 are presented along with their characteristic forces and the conditions of the experiments. The mean characteristic force and the standard deviation represent the primary and secondary observed cracks, which correspond to the first and second peaks in the force-displacement curves presented in Figure 4. To better emphasise the magnitude of scatter, the relative standard deviation is also indicated for the primary cracks characteristic forces. Although in most of the tests, more than one secondary crack occurred, only the ones that appeared first are presented, since the force distribution of the other cracks produced a significant dispersion. Batches 1 through 7 were used to optimise the results by decreasing the standard deviation. The manufacturing process of the mortar was improved in Batches 1-4, whereas the effect of the loading speed was tested in Batch 3. In Tests 5 and 6, wooden support blocks were added to the device to minimise the small rotations of the disk suspected to be causing the high standard deviation, and Test 7 corresponded to the first experiments done with the DIC.

The characteristic forces of the diametric tests varied between 5.80 and $7.36 \mathrm{kN}$. This variation, which could be caused by slight differences in the curing process, remained within an acceptable range (a standard deviation smaller than $0.5 \mathrm{kN}$ ).

Like all brittle and quasi-brittle failures of cement based materials, the behaviour at failure of our specimens under the configurations studied is expected to be dependent of their size. This size effect has been extensively studied in the case of diametric splitting tests, both experimentally and numerically, and was shown to be correctly described by the law proposed by Bažant (1984). It can be deduced that the critical force associated with the primary crack is equally affected by the size effect since the primary fracture follows the same tensile mode as Brazilian type splitting tests. As for the secondary cracks that were shown to propagate following a mode II, Bažant and Sun (1987) showed that the strength of specimens under shear failure also exhibits a considerable size effect, which could be modelled using the same law (Bažant, 1984).

The comparison between the characteristic forces of the configurations with 2 contacts (diametric compression), 4 contacts (4-90, 4-45 and 4-90-60), and 5 contacts 


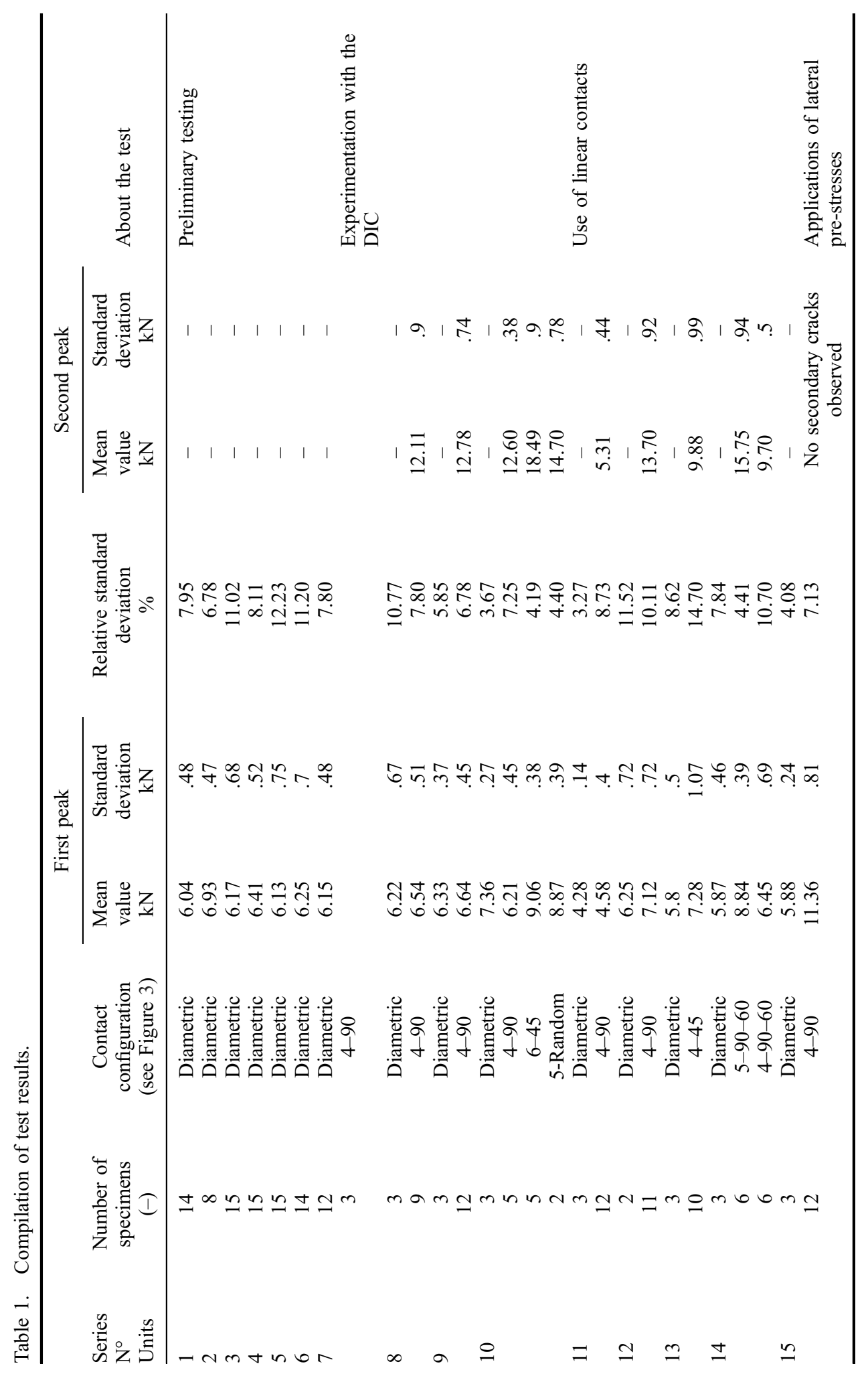




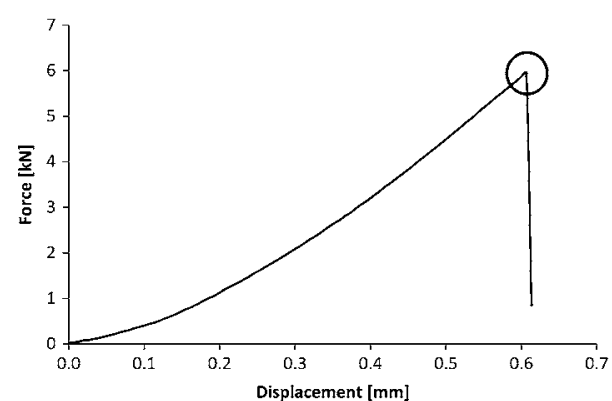

(Diametric)
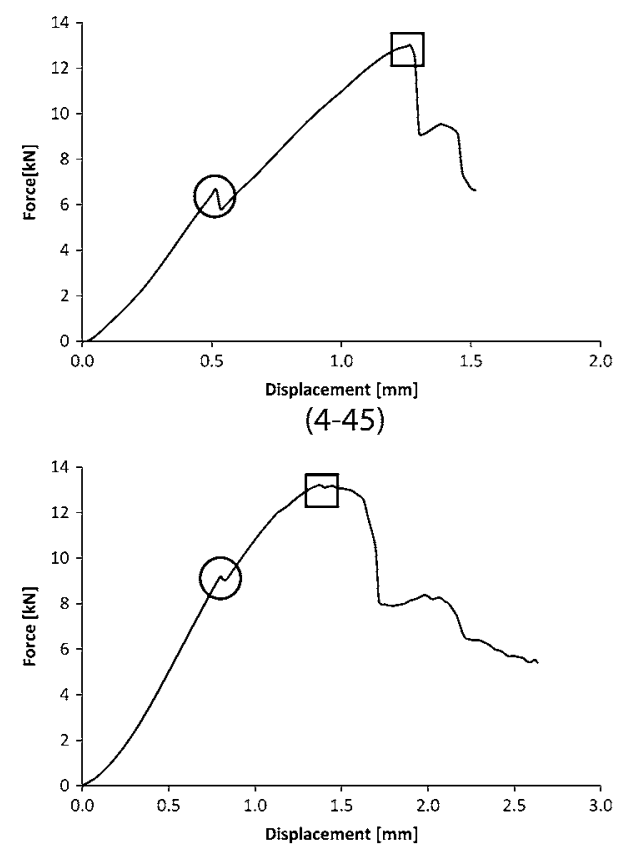

$(5-90-60)$

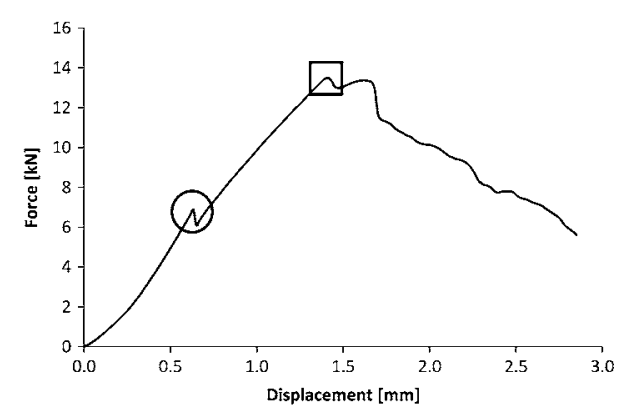

(4-90)

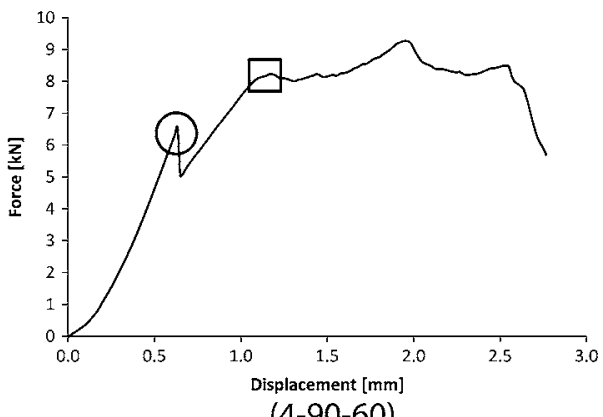

$(4-90-60)$

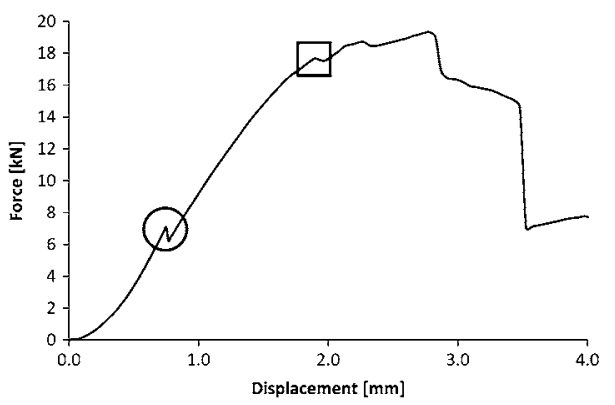

$(6-45)$

Figure 4. Typical force displacement curves associated to different configurations. The diametric cracks are represented by circles whereas the secondary cracks are represented by squares.

(5-90-60) confirms that a higher coordination number results in a higher characteristic force. The exception represented by the 6-45 test can be noted, for which the critical force was found higher than the ones corresponding to the tests with 4 contacts, but lower than the force corresponding to the 5-90-60 test. This shows the importance of the contact position on the crushing behaviour of particles. It can also be noted that the presence of diametrically opposed sets of forces increases the characteristic force of the first crack, as deduced from a comparison between the 4-90 and the 4-90-60 configurations. Therefore, it is confirmed that the contact positions along the lateral surface highly affect particle crushing. The critical force of the secondary cracks depends on the length of the crack path: secondary cracks that follow longer paths have the lowest critical forces in comparison to the ones with shorter paths. This can be seen by comparing the tests 4-90, 4-45 and 4-90-60, all with a coordination number of 4 , but with 
different secondary crack paths. The secondary crack in the 4-90 configuration has the shortest path, and its characteristic force is significantly higher than the ones linked to the 4-45 and 4-90-60 tests where the secondary cracks follow longer paths. The secondary forces are equally affected by the coordination number and position.

Typical force displacement curves of the tested configurations are presented in Figure 4. The sudden drops in the forces correspond to the characteristic force with regard to a rupture event.

The validity of Equations (1) and (2) to estimate the effect of the coordination number on the critical force of failure of a particle has been examined in light of the experimental results obtained. The use of Equation (1) requires the definition of the factor $f_{\mathrm{D}}$ that represents the effect of curvature of the grains on the fracture.

The following form for the curvature factor is proposed by Sukumaran et al. (2006), based on the analytical solution of Timoshenko and Goodier (1961) for an elastic disk particle compressed by disks instead of platens:

$$
f_{D}=\frac{(D=l)}{(D=l)+1}
$$

With $D$ being the diameter of the surrounding particles. It should be noted that this equation captures the effect of the roundness of the surrounding particles, and is then different from Bažant's size effect law (1984), which represents the evolution of the critical stress with the size of the particle.

The work of Timoshenko and Goodier doesn't examine crushing under planar surfaces. In addition, the crushable particle and the surrounding particles are supposed to have the same elastic properties. For the purpose of this numerical study, only the surface contacts achieved by the surface type clamping bars are considered. In this case, $D \rightarrow+\infty$, which means that $f_{D} \rightarrow 1$. A value of $\sigma_{C}$ is deduced from the fitting of the calculations with the experimental results, achieved by minimising an error function defined as the sum of the absolute values of the differences between the experimental and the calculated critical forces of the configurations considered. Figure 5 shows

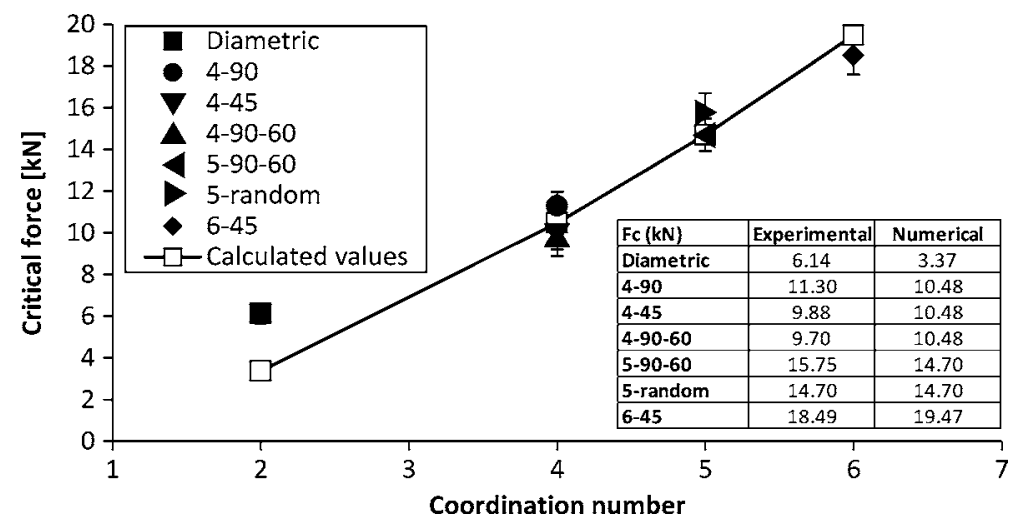

Figure 5. The experimental values of the critical force plotted with the values predicted by Equation (1). 
a good correspondence between the experimental values of the mean critical force and the values computed using the formulas (1), (2) and (3).

\section{The effect of a secondary pre-stress}

Tests on samples from Batch $n^{\circ} 15$ are presented in Table 1. They were performed in order to investigate the effect of a secondary lateral pair of active contacts forces on particle crushing by applying a preliminary stress on the specimens. Twelve tests in the 4-90 configuration were conducted, where the two lateral tightened contacts could represent the effect of the contact forces due to surrounding particles within a strong force chain. The bolts of the clamping bars were tightened carefully to ensure reasonably comparable results, because no force was measured. A typical force displacement plot is presented in Figure 6. If we compare this figure to the one corresponding to a normal 4-90 test (Figure 4), we can see that only one peak was obtained, which corresponds to one diametric crack. No secondary cracks were observed; instead, the specimen broke into multiple fragments, which explains the post peak ductile-like failure in the corresponding curves of Figure 7. As for the 4-90 test with passive contacts, the loading continued after the final crack, which resulted in the comparable ductile-like post peak behaviour (the fragments were held together by the surface contacts, allowing the specimen to withstand the loading). The mean value of the critical force for the pre-stressed test was $11.36 \mathrm{kN}$, which is higher than all the critical forces corresponding to the first crack of the tested configurations, and especially higher than the mean $7.12 \mathrm{kN}$ corresponding to the 4-90 test without a pre-stress.

\section{The effect of the contact type}

In the specimens of Batch 11, linear contacts were used instead of surface contacts to study the effect of the contact type on the rupture of the specimen. The contacts along the loading axis and the ones along the loading surface were both modified (Figure 2). The fracture patterns and the resulting fragments were not affected by the contact type. Three diametric compression tests, and 12 crushing tests in the 4-90 configuration were

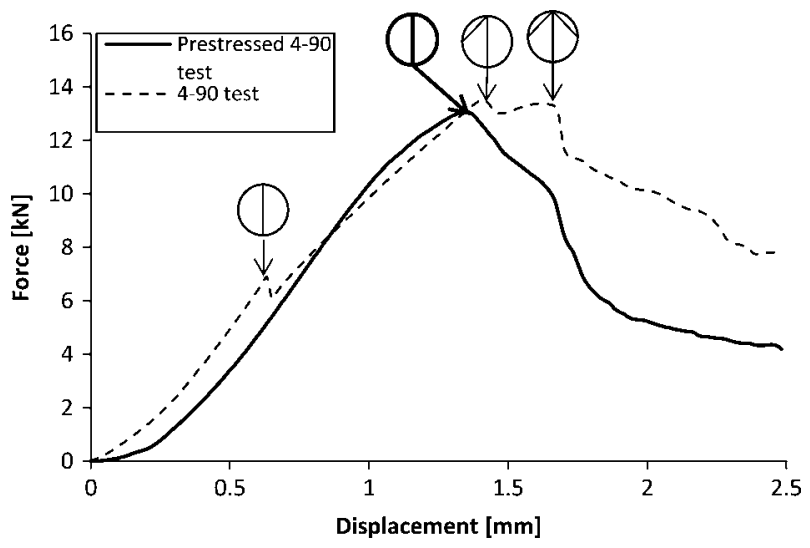

Figure 6. Typical force displacement curve of the pre-stressed 4-90 tests. 


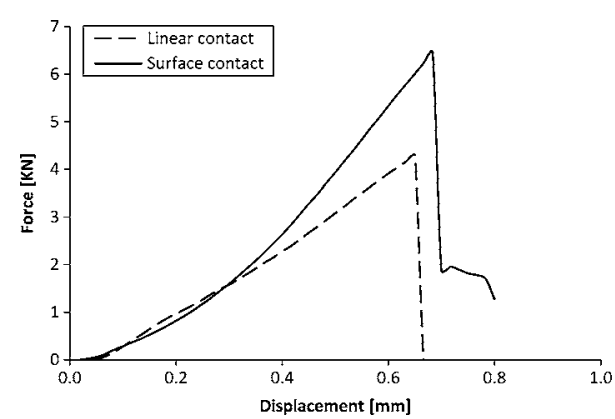

(a)

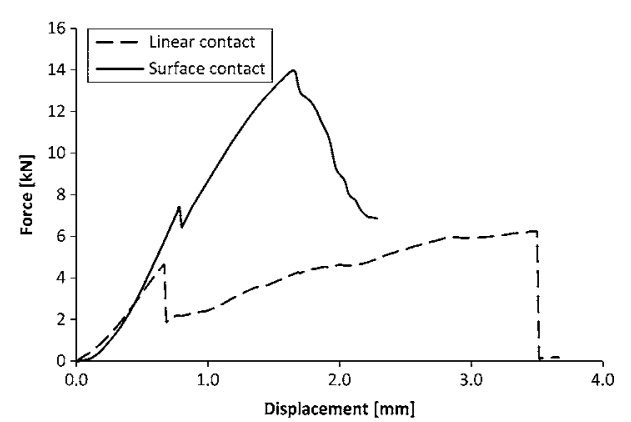

(b)

Figure 7. Force displacement curves for (a) Diametric compression and (b) 4-90 configuration with different contact types (Salami et al., 2015).

conducted. The results were compared with the results of samples from Batch 12, where surface contacts were used. Typical force displacement curves are presented in Figure 7.

It can be noted that the cracks occurred at higher forces with surface contacts rather than with linear contacts. This is true for the primary cracks in the diametric and the 4-90 configurations, and for the secondary cracks in the 4-90 configuration. In case of a diametric compression, the force decreased from 6.25 to $4.28 \mathrm{kN}$. This can be explained by the higher concentration of forces near the contacts in the case of a contact along a line. Indeed, both the contact types are non-conforming (the area of contact is smaller than the characteristic dimensions of the contacting bodies, which means that at least one of the two bodies deforms when a contact area appears), and the surface contacts allow the load to spread over a wider area. It should be noted that the load concentration near the contacts leads to both primary and secondary cracks, as will be shown below. The same reasoning could be extended to the secondary cracks observed in the 4-90 configuration, since they both result from a concentration of stresses near the contacts, even if the fracture mechanism is different. A difference in the post peak failure between the two 4-90 tests was also noted. This may have been caused by the higher adherence of the particle to the contacts favored by the high friction area of the surface types. The specimen was held together after fracture, and continued to carry the applied load, causing an increasingly higher damage, and resulting in the observed behaviour. If the specimen was given enough time to fail after the second crack occurred, it usually broke into multiple small fragments. The linear contacts, however, penetrated within the cracked space, causing a sudden failure.

\section{Fragmentation process}

After conducting the tests, fracture paths and fragmentation patterns of the disks were observed. In all cases, a central crack appeared along the diametric loading axis. Other cracks, referred to as secondary cracks, appeared later on. For each contact configuration, the test resulted in a specific set of fragments. Although the fragments were not always identical, they appeared to follow the same pattern. If we take the example of the 4-90 test configuration presented in Figure 8, cracking occurred throughout the two top circular segments. Other tests in the same configuration resulted in the fragmentation of one or more of the four segments linking the contacts of the particle. For the other 


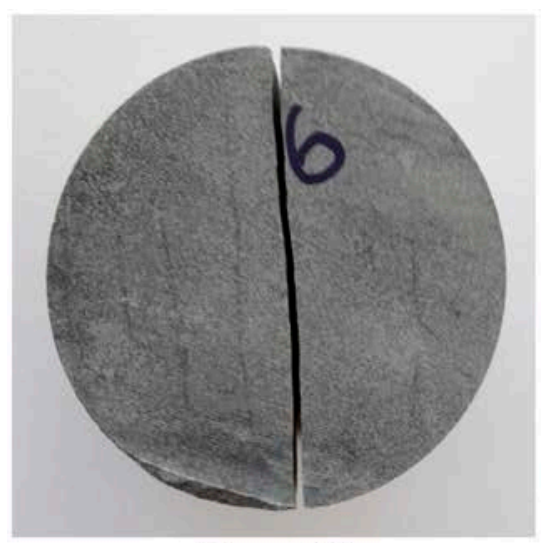

(Diametric)

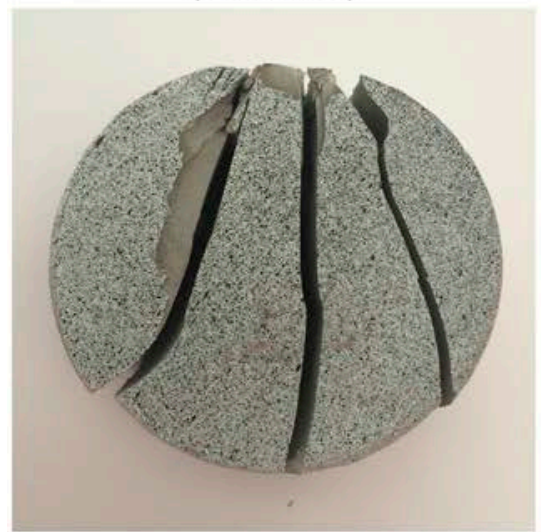

$(4-45)$

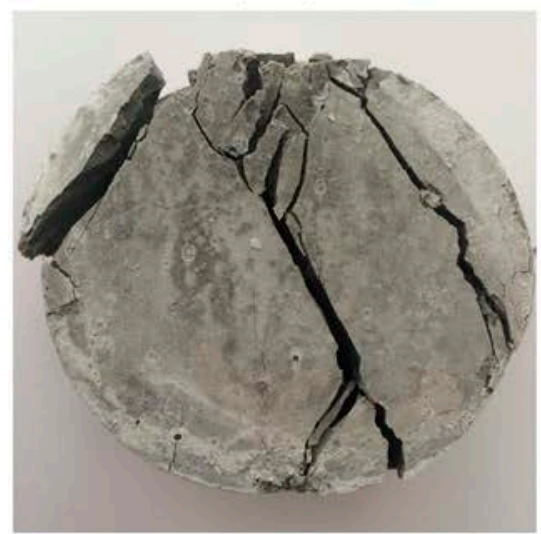

$(5-90-60)$

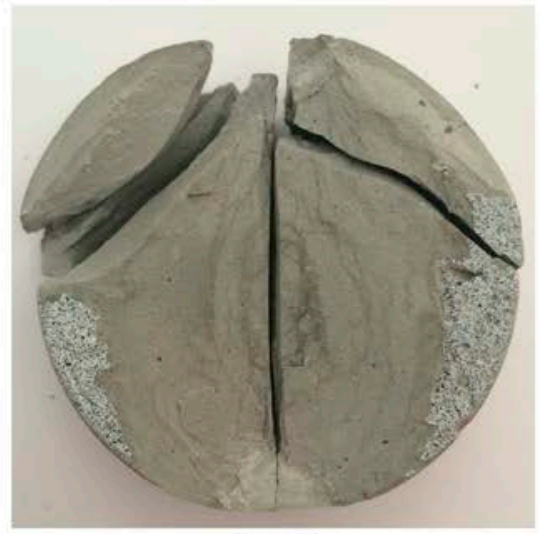

$(4-90)$

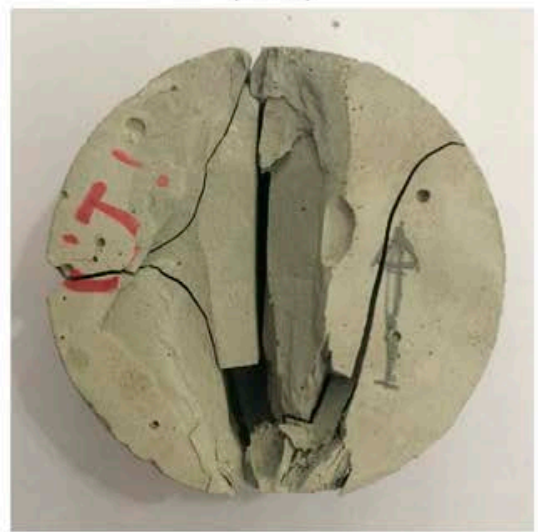

$(4-90-60)$

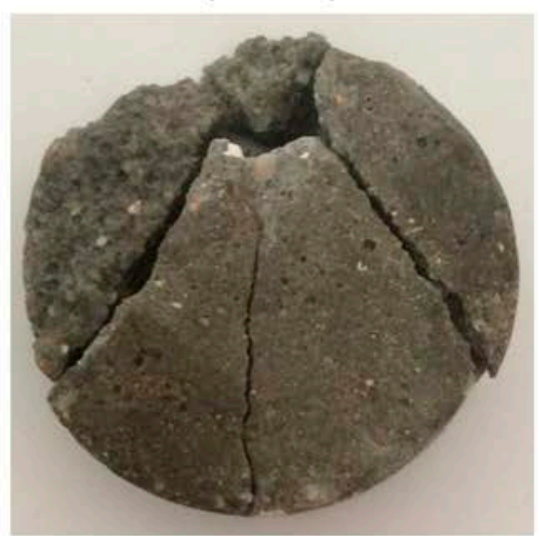

$(6-45)$

Figure 8. Typical fragmentation patterns corresponding to each tested configuration.

configurations, the cracks appeared to always follow a predetermined path imposed by the configuration. The secondary cracks originated near one of the contacts, and propagated towards the farthest load bearing contact (on the top or the bottom). 
The specimens of Figure 8 were chosen out of the other specimens because at least two secondary cracks were clearly observed. In order to achieve these results, the forces were allowed to reach higher values, which explain the additional small fragments observed (in 5-90-60 and 6-45). These fragments are the result of the failure of the contact area, and do not appear in other tests.

The predominant loading along the vertical axis leads to an initial appearance of a diametric crack. The explanation for this phenomenon can be found in the literature illustrated by a volume fracture model (Tsoungui, Vallet, Charmet, et al., 1999): a traction zone develops near the centre of the specimen, and leads to the formation of a crack that propagates along the loading axis, ultimately breaking the specimen in half. However, other authors disagree with the volume fracture model and suggest that in a diametric compression test, and in the case of non-conforming contacts, the fracture originates near the contacts, and propagates along the loading axis, causing the specimen to break in half. Wang and Xing (1999) studied the case of a Brazilian test with conforming contacts, and determined a minimum loading angle (angle from the centre of the disk to the edges of the contact surface) for the crack to originate near the centre of the grain. A specimen with flattened edges, or arch-arch tests such as the ones suggested by the ISRM (International Society for Rock Mechanics, 1978) should be able to reproduce the volume fracture model, since the contact area is large, and the load is spread along this surface. In our case, given that the contacts are non-conforming, the fracture is expected to start from the contacts. Another cracking mechanism, suggested by (Tsoungui, Vallet, Charmet, et al., 1999) and termed the contact fracture model, was used to study grains impacting a rigid wall. Under this model, a compressive strain propagates from the contact point through the specimen at the moment of the impact. This model seems to reproduce the primary cracking observed in our tests better than a volume model. To the best of our knowledge, no literature is available on the secondary cracks and their mechanisms of propagation other than the fracture criterions presented in the introduction.

To better understand the mechanics of the observed fractures, a series of tests in the diametric, 4-90, 6-45 configurations were monitored by DIC. The results for the diametric and 4-90 tests are summarised in Figure 9. The cracks in the 6-45 configuration followed the same pattern as in the 4-90 configuration. The extension and shear strain distributions along the surface allowed us to draw several conclusions. For the diametric compression, the cracks appeared to originate near the contacts and propagated towards the centre. This was also the case for the primary crack in the other tests. The secondary cracks originated near the contacts, and propagated towards the farthest load bearing contact, as shown in the fragmentation of the 4-45, the 4-90-60, the 5-90-60, and the 6-45 configurations. The newly formed half disk fragment is under the effects of a secondary contact and the opposed load bearing contact, either top or bottom. This resulted in the crack propagating in a shear mode towards the top or bottom contact. Consequently, the shear strains were highly concentrated in these cracks paths, suggesting that the fracture occurred though a shear mode (mode II), as suggested by Ben-Nun and Einav (2010). The high shear strains along the diametric axis were caused by friction, as the specimen was already fractured in half when the secondary crack was observed.

It is also possible to analyse the crack propagation based on the distributions of the principal strains. Figure 10 shows the directions and magnitudes of the principal strains inside the disk before and during the propagation of the longitudinal fracture, and during the propagation of the secondary fracture. The red lines correspond to the principal extensions while the blue lines represent the principal compressions. The length of each 


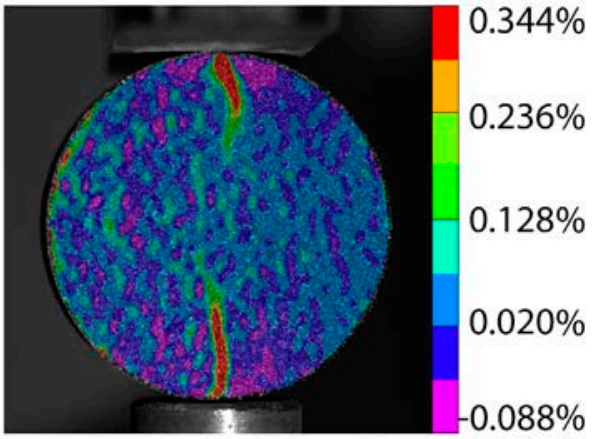

(a)

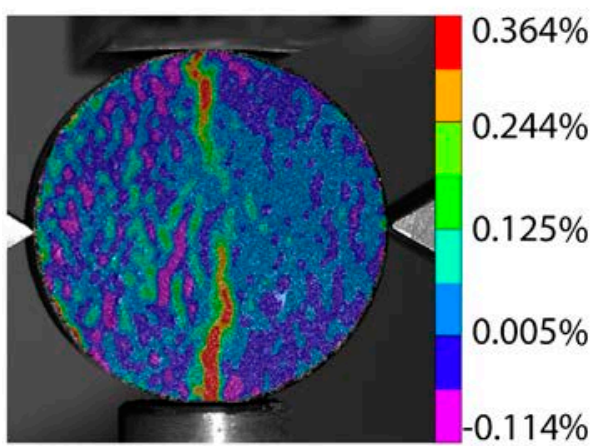

(c)

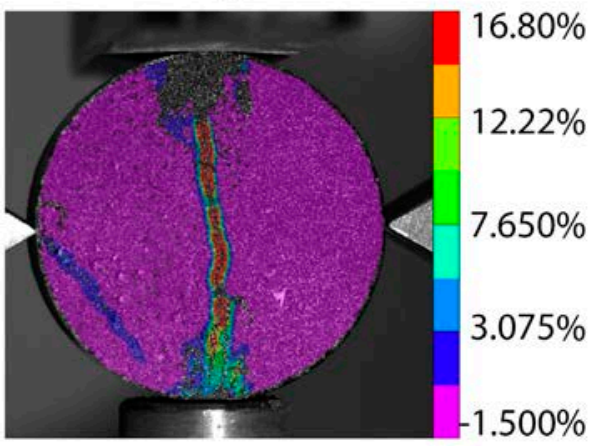

(e)

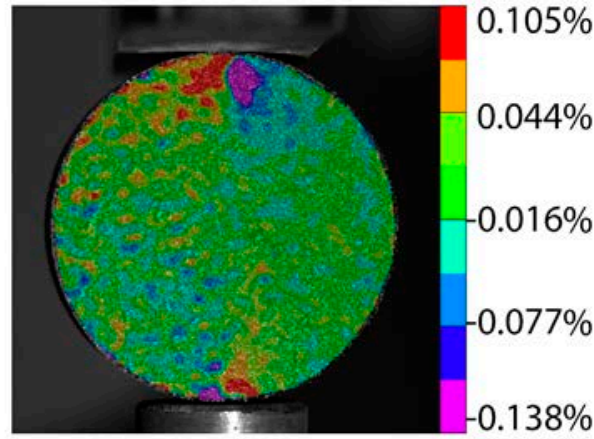

(b)

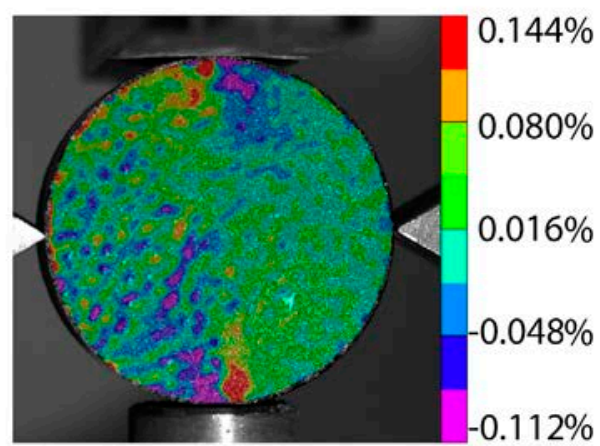

(d)

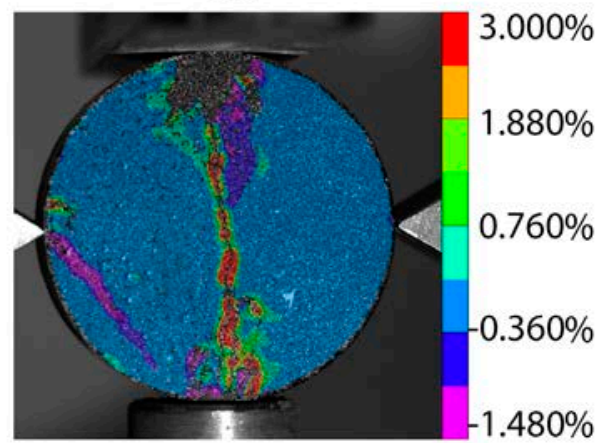

(f)

Figure 9. Distribution of the strains along the $x$ axis $\left(\varepsilon_{x x}\right)$ and the shear strains $\left(\varepsilon_{x y}\right)$ prior to fracture. For the diametric compression: (a) strains along the $x$ axis and (b) shear strains. For the 4-90 configuration: (c) strains along the $x$ axis prior to the first crack, (d) shear strains prior to the first crack, (e) strains along the $x$ axis prior to the second crack, (f) shear strains prior to the second crack.

line is proportional to the magnitude of the corresponding principal strain. However, the different images presented in Figure 10 are plotted at different scales. In image (a), it is clear that the directions of the principal extension are perpendicular to the direction of the crack propagation. It can be concluded that the longitudinal crack is caused by traction, which corresponds to an opening mechanism of cracking (mode I). 


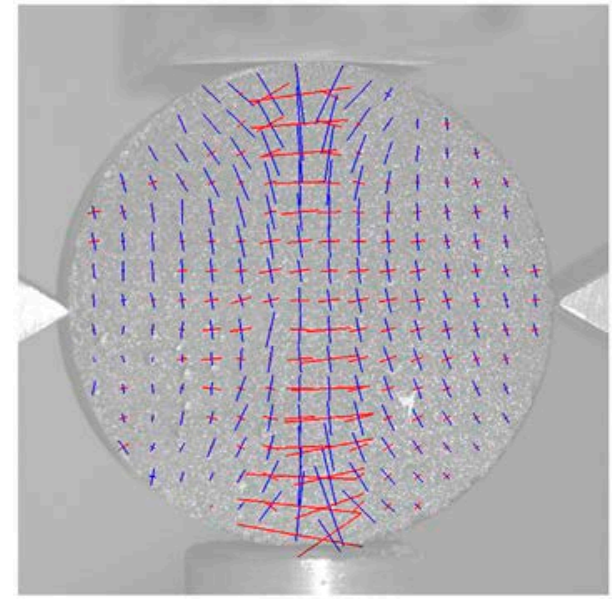

(a)

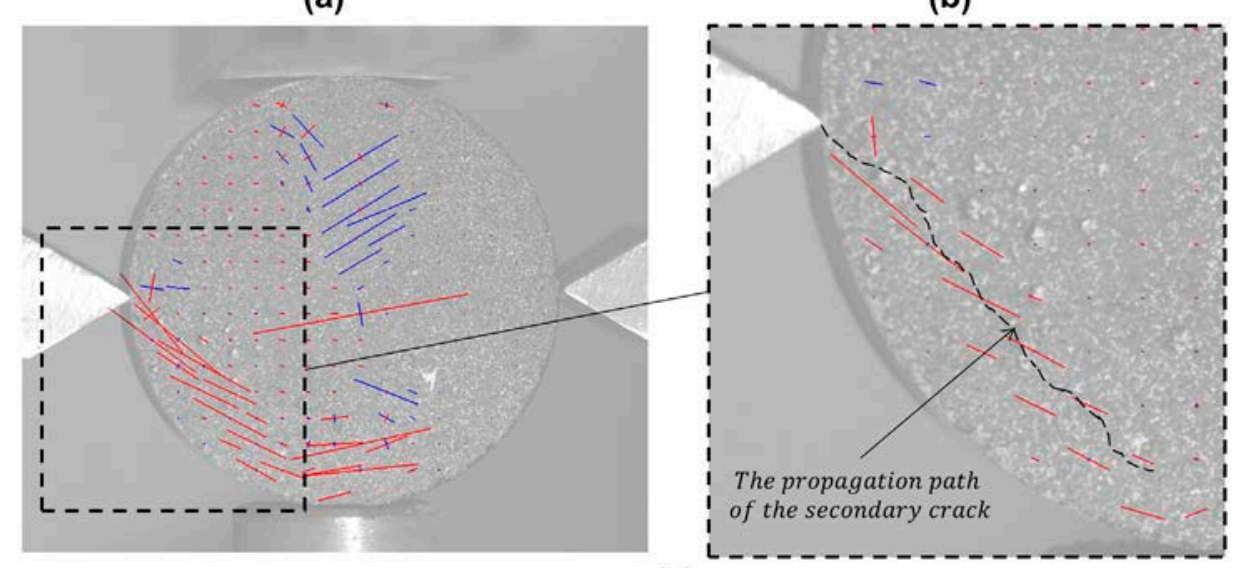

(c)

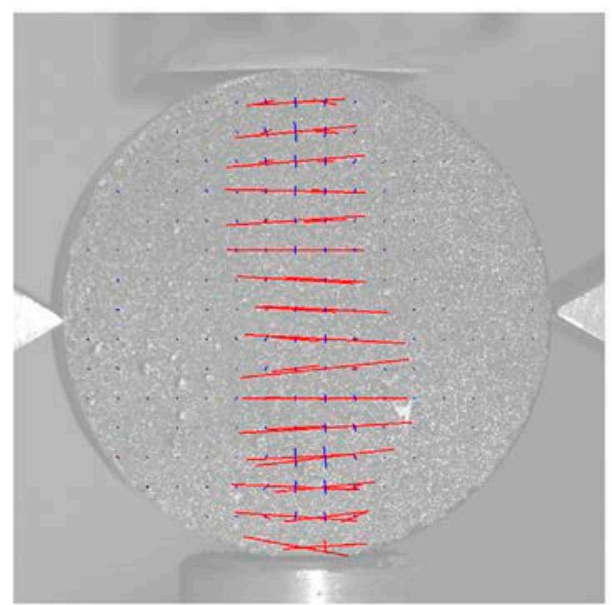

(b)

Figure 10. Principal strain distributions: in red, the principal extensions; in blue, the principal compressions. (a) Before the primary fracture, (b) at the moment of the primary fracture, (c) at the moment of the secondary fracture.

As for the secondary cracks, Figure 9 showed a concentration of shear strains in the fracture zone, which allows us to conclude that this mode of crack propagation is in-plane shearing (mode II). An analysis of Figure 10(c) allows us to confirm this conclusion. In the zone where the secondary crack develops and prior to cracking, the extensions are almost parallel to the path of the future crack. The crack should then propagate according to a mixed mode I and II, with a predominance of mode II.

Out-of-plane shearing (which corresponds to mode III) cannot be identified from our data, since the DIC performed is planar. Stirling, Simpson, and Davie (2013) used 3D DIC on diametric compression tests, and showed that out-of-plane displacements do occur near the two contacts, and are especially more pronounced when the contacts between the specimen and the loading device are non-conforming. 


\section{Conclusion}

The results presented in this paper agree with several of the previous numerical and theoretical works on the subject, although the aim of the fracture models in the literature has not been to reproduce the behaviour studied by our tests. It has been shown that the contact numbers and positions play an important role in the fragmentation of an individual grain. DIC was used to measure the strains along the surface of the disk, which provided insight on the initiation of the cracks and its propagation path. Two types of cracks could be distinguished, each corresponding to a different crack mode and dependent on the contact arrangement. The effect of the contact type was studied and the results showed that the critical force depended strongly upon the contact area.

One of the many applications of these results could be the improvement of DEM models considering crushable materials. Indeed, most of the problems faced in modelling grain breakage concern the fracture initiation condition, the fragment number and size. A new model based on the coordination number may be considered.

These preliminary results are the beginning of an in-depth experimental study on the effects of the coordination number on grain crushing. The effect of the contact forces, which was briefly tested in this study, will in the future be fully investigated using springs for the lateral contacts, or by measuring the contact forces in the lateral bars by using strain gauges.

\section{Disclosure statement}

No potential conflict of interest was reported by the authors.

\section{References}

Auvinet, G., \& Marsal, R. (1975). Statistical model of grain breakage. In: Proceedings, Fifth Pan-American Conference on Soil and Foundation Engineering (Vol. 1, pp. 193-204). Buenos Aires, Argentina.

Bažant, Z. P. (1984). Size effect in blunt fracture: Concrete, rock, metal. Journal of Engineering Mechanics, 110, 518-535.

BaŽant, Z. P., \& Sun, H. H. (1987). Size effect in diagonal shear failure: Influence of aggregate size and stirrups. ACI Materials Journal, 84, 259-272.

Ben-Nun, O., \& Einav, I. (2010). The role of self-organization during confined comminution of granular materials. Philosophical Transactions of the Royal Society A: Mathematical, Physical and Engineering Sciences, 368, 231-247.

Blaber, J., Adair, B., \& Antoniou, A. (2015). Ncorr: open-source 2D digital image correlation matlab software. Experimental Mechanics, 55, 1105-1122.

Correlated Solutions. (2009). Vic-2D testing guide. Retrieved from http://correlatedsolutions.com

International Society for Rock Mechanics. (1978). Suggested methods for determining tensile strength of rock materials. International Society for Rock Mechanics Commission on Standardization of Laboratory and Field Tests, 15, 99-103.

Jaeger, J. C. (1967). Failure of rocks under tensile conditions. International Journal of Rock Mechanics and Mining Sciences \& Geomechanics Abstracts, 4, 219-227.

McDowell, G. R., \& Bolton, M. D. (1998). On the micromechanics of crushable aggregates. Geotechnique, 48, 667-679.

McDowell, G. R., \& Amon, A. (2000). The application of Weibull statistics to the fracture of soil particles. Soils and Foundations, 40, 133-141.

McDowell, G. R., Bolton, M. D., \& Robertson, D. (1996). The fractal crushing of granular materials. Journal of the Mechanics and Physics of Solids, 44, 2079-2101.

NF EN 196-1. (2006). Méthodes d'essais des ciments - Partie 1 : détermination des résistances mécaniques [Methods of testing cement - Part 1: determination of strength]. Saint-Denis La Pleine: Afnor. 
Salami, Y., Dano, C., Hicher, P.-Y., Colombo, G., \& Denain, P. (2015). The effects of the coordination on the fragmentation of a single grain. IOP Conference Series: Earth and Environmental Science, 26, 012015.

Stirling, R. A., Simpson, D. J., \& Davie, C. T. (2013). The application of digital image correlation to Brazilian testing of sandstone. International Journal of Rock Mechanics and Mining Sciences, 60, 1-11.

Sukumaran, B., Einav, I., \& Dyskin, A. (2006). Qualitative assessment of the influence of coordination number on crushing strength using DEM. In: 5th World Congress on Particle Technology (pp. 1-8). Orlando, FL.

Timoshenko, S. P., \& Goodier, J. N. (1961). Théorie de l'élasticité [Theory of elasticity]. Béranger. New york: McGraw-Hill.

Todisco, M. C., Coop, M. R., \& Guo, Q. (2015). The effect of the coordination number on particle crushing. In K. Soga, K. Kumar, G. Biscontin, \& M. Kuo (Eds.), Geomechanics from Micro to Macro (pp. 1063-1068). London: Taylor \& Francis.

Tsoungui, O., Vallet, D., \& Charmet, J.-C. (1998). Use of contact area trace to study the force distributions inside 2D granular systems. Granular Matter, 1, 65-69.

Tsoungui, O., Vallet, D., \& Charmet, J.-C. (1999). Numerical model of crushing of grains inside two-dimensional granular materials. Powder Technology, 105, 190-198.

Tsoungui, O., Vallet, D., Charmet, J.-C., \& Roux, S. (1999). Size effects in single grain fragmentation. Granular Matter, 2, 19-27.

Wang, Q.-Z., \& Xing, L. (1999). Determination of fracture toughness KIC by using the flattened Brazilian disk specimen for rocks. Engineering Fracture Mechanics, 64, 193-201. 